\title{
The breakup of levitating water drops observed with a high speed camera
}

\author{
C. Emersic and P. J. Connolly \\ School of Earth, Atmospheric and Environmental Sciences, University of Manchester, Manchester, UK \\ Received: 6 April 2011 - Published in Atmos. Chem. Phys. Discuss.: 15 April 2011 \\ Revised: 19 September 2011 - Accepted: 27 September 2011 - Published: 11 October 2011
}

\begin{abstract}
Collision-induced water drop breakup in a vertical wind tunnel was observed using a high speed camera for interactions between larger drop sizes (up to $7 \mathrm{~mm}$ diameter) than have previously been experimentally observed. Three distinct collisional breakup types were observed and the drop size distributions from each were analysed for comparison with predictions of fragment distributions from larger drops by two sets of established breakup parameterisations. The observations showed some similarities with both parameterisations but also some marked differences for the breakup types that could be compared, particularly for fragments $1 \mathrm{~mm}$ and smaller. Modifications to the parameterisations are suggested and examined. Presented is also currently the largest dataset of bag breakup distributions observed. Differences between this and other experimental research studies and modelling parameterisations, and the associated implications for interpreting results are discussed. Additionally, the stochastic coalescence and breakup equation was solved computationally using a breakup parameterisation, and the evolving drop-size distribution for a range of initial conditions was examined. Initial cloud liquid water content was found to have the greatest influence on the resulting distribution, whereas initial drop number was found to have relatively little influence. This may have implications when considering the effect of aerosol on cloud evolution, raindrop formation and resulting drop size distributions. Calculations presented show that, using an ideal initial cloud drop-size distribution, $\sim 1-3 \%$ of the total fragments are contributed from collisional breakup between drops of 4 and $6 \mathrm{~mm}$.
\end{abstract}

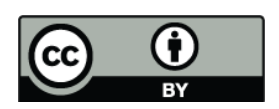

Correspondence to: C. Emersic (christopher.emersic@manchester.ac.uk)

\section{Introduction}

Raindrop breakup events are important to the evolution and formation of drop size distributions in precipitating clouds, particularly for warm rain processes in which frequent collision, coalescence and breakup events play a major role in the production of raindrop-sized precipitation. To investigate the nature of this phenomenon, researchers have performed experimental laboratory studies to observe breakup events, modelling simulations of the evolution of cloud drop size distributions based on parameterisations from the experimental observations, and compared results to observations of drop size distributions in natural clouds. A short review of the aspects of these areas of research relevant to this study is presented.

Very few experimental laboratory investigations have been conducted to measure the drop size distributions resulting from collision-induced breakup. To our knowledge to date, these studies include McTaggart-Cowan and List (1975a), Low and List (1982a), and Barros et al. (2008), and all three have employed an experimental setup which involved colliding falling drops. McTaggart-Cowan and List (1975a) and Low and List (1982a) used a drop acceleration system in which drops created at two sources, vertically accelerated toward terminal velocity as they fell. A small blower was used to horizontally deflect one drop into the path of another to encourage collision; the larger, faster-falling drop always collided from above onto the smaller slower-falling drop. The resulting coalescence-breakup event was observed using a camera, which allowed drop fragments to be counted afterwards. Drop sizes were reproducibly controlled, and 10 drop-pair size combinations were used, ranging in diameter from $0.395-4.600 \mathrm{~mm}$. A more detailed discussion of their experimental apparatus is given in McTaggart-Cowan and List (1975b). Of 25000 drop collisions, it was reported that only 712 resulted in breakup, with the breakup type

Published by Copernicus Publications on behalf of the European Geosciences Union. 
distribution consisting of $27 \%$ filament (a.k.a. neck), $55 \%$ sheet, $18 \%$ disc, and only 3 events as bag breakup (these breakup types are defined below). The breakup type that was more likely to occur seemed to be dependent on the kinetic energy of the collision event, the impact location, and the angle between the two colliding drops.

Filament (or neck) breakup describes when a connecting bridge of water forms between the two separating drops after collision (usually a glancing collision). As the drops separate further, this bridge disintegrates into a series of smaller drops; the two parent drops retain much of their original mass. Sheet breakup occurs when the collision is less glancing and more direct, with the strike point on the edge rather than the centres of the drops. The impact energy causes the drops to spread preferentially at one side to form a sheet. The original smaller drop becomes indistinguishable in the resulting mass, and the sheet breaks up into many smaller drops and a remaining larger drop. Disc breakup is a variation of sheet breakup and occurs when the impact point is more central. The smaller drop, effectively impacting from underneath the larger drop, coalesces and causes the whole drop to spread out flat into a disc. The thinning edges lift due to aerodynamic drag and disintegrate into many smaller drops; the parent drops are often indistinguishable in the resulting drop distribution. Bag breakup was rarely observed and involved the flattening out of the resulting drop after collision as with disc, but the centre lifts and inflates to form a thin bag which bursts explosively to produce many small drops.

Barros et al. (2008) used a similar experimental setup which again encouraged two vertically falling drops to interact. They interacted 6 drop-pair size combinations with drop sizes ranging from $0.93 \mathrm{~mm}$ to $4.8 \mathrm{~mm}$ and found that in 535 collision events, 142 were filament (27\%), 47 were sheet $(9 \%)$ and 58 were disc $(11 \%)$.

Many of the researchers who have conducted laboratory experiments have subsequently parameterised their observations and examined the accuracy of these parameterisations relative to the observations of others and within various cloud simulations. Low and List (1982b) were the first to form numerical parameterisations for filament, sheet and disc breakup types based on their initial observations. McFarquhar (2004a) later reformulated parameterisations which took into account mass conservation, provided a more physical basis for extending the results of the original 10 colliding pairs to arbitrary drop pairs, and had a complete uncertainty analysis. These parameterisations formed the standard to which all subsequent research made comparisons and to which this study compares.

Barros et al. (2008) constructed a computer model based on the Low and List parameterisations and compared the results for the drop-pair sizes they used with the output. They found that the simulated location and width of peaks in the fragment size distributions showed good agreement, but predicted greater concentrations of drops of size $0.5 \mathrm{~mm}$ or larger and fewer concentrations of drops of size $0.2 \mathrm{~mm}$ or smaller than were observed in their experiments.

McFarquhar (2004b) found from modelling studies that the variation in raindrop size distributions depends heavily on the drop distribution at precipitation onset, and that the large spread in the breakup distributions can account for the inability of equilibrium distributions to form in nature. Clustering of raindrops, where larger, faster falling drops catch up with smaller, slower falling drops during descent, was also found to increase the chances of interactions between drop-pairs. Modelling studies examining how the drop size distributions in rain-producing clouds evolve over time have found that three-peaked distributions can form (McFarquhar and List, 1991a, b; List and McFarquhar, 1990). In their 1-D simulations, multiple pulses of rain with durations of between 2$10 \mathrm{~min}$ at a repeated rate of every $4-12 \mathrm{~min}$ at the top of the shaft led to drops arriving in packages at the ground, with the largest drops arriving first in each package. Averaged over time, the three-peak distributions were observed. Simulations in which clustering and associated raindrop overlapping was prevented did not produce the three-peak distribution, and it was proposed that the drop interactions from their differing terminal velocities were necessary for subsequent coalescence and breakup events to give rise to the distribution peaks. Simulations using the newer parameterisations by McFarquhar (2004a) revealed a three-peak steady-state distribution of raindrops is no longer realized in the numerical output. Instead, a two-peak distribution is realised, with the two peaks representing the drops generated by coalescence and by the breakup fragments respectively.

A number of observations have measured multiple drop distribution peaks at similar sizes to those modelled (Debeauville et al., 1988; Steiner and Waldvogel, 1987; Willis, 1984; Zawadzki and Antonio, 1988); however, some observational studies have noted drop size distributions without multiple modes or with time- and altitude-varying single modes (Joss and Gori, 1978; Warner, 1969). However, much of the original observational evidence of the threepeak raindrop distribution is now in question. McFarquhar and List (1993) showed that the effects of irregularities in the diameter classification of the Joss-Waldvogel disdrometer produced artificial peaks at the locations where peaks had been reported in observational studies. Furthermore, the magnitudes of the instrument related peaks were similar to the magnitudes of the observed peaks, negating some of the previous evidence of three-peak distributions.

In this study, larger drops than those used by other experimental researchers were investigated. The spontaneous (a.k.a. aerodynamic) breakup of larger drops may have some significance to drop spectrum evolution. In general however, spontaneous drop breakup is not considered to be as influential to the resulting raindrop-size distribution in warm rain processes owing to difficulties in large drops forming without prior collisionally-induced breakup having already occurring (Magarvey and Geldart, 1962; Low and List, 1982a). 
Nevertheless, Hobbs and Rangno (2004) conducted airborne measurements beneath cumulus congestus clouds formed in one case by a biomass fire in Brazil, and in another case, in very clean but atmospherically unstable conditions in the Marshall Islands in the tropical Pacific Ocean, and observed very large raindrops of the order of $1 \mathrm{~cm}$ in diameter in their associated rainshafts. These drops were thought to have formed rapidly by the coalescence of drops in narrow regions of the cloud where liquid water contents were unusually high. It is also suspected that large drops can form in the regions between updraughts where drop-drop collisions (and therefore breakups) are minimised but that scavenging of smaller cloud droplets can lead to drop growth to $\sim 8 \mathrm{~mm}$ as observed by Beard et al. (1986).

Using a wind tunnel to generate an air well, it is possible to levitate and interact drops injected into the air stream from above. Drop-pairs of larger sizes than have been used before (up to the sizes observed in nature as discussed above) have been interacted, and the breakup events filmed using newly-available high speed video technology. The primary objective of this study was to examine the droplet size distributions that result after a collision-induced breakup occurs, and use our observations to evaluate existing parameterisations of collision-induced break-up over a different range of drop sizes than has been done previously. It was not our objective to examine the conditions that lead to breakup of a particular type. A computer model based on the parameterisations of Low and List (1982b) and McFarquhar (2004a) is used to predict expected results for the larger drop-pair sizes used, and help verify extrapolations from the parameterisations. Furthermore, simulations of cloud drop-size distribution evolution and contributions to this from larger drop-pair sizes are performed to examine the significance of these interactions. A description of the experimental procedure is presented in Sect. 2, a discussion of experimental observations and the results of computer simulations are presented in Sect. 3, and a concluding summary is given in Sect. 4.

\section{Experimental setup and procedure}

Experiments were conducted using a vertical wind tunnel (Fig. 1). Air is passed through a settling chamber before passing out through an upper orifice covered by a wire grate to produce a radial velocity profile from the centre where the air speed is lowest. The presence of an upper plate provides a back-pressure, which combined with the radial velocity profile, generates a stagnation pressure well in which injected drops can levitate. Additional drops can then be injected into the air stream from above which would find their way into the well and may interact with any drop present. The airspeed as a function of radius in the well at approximately the height where the drops vertically oscillate for the given air speed (i.e. the part of the system where drop interactions took place) was measured using a calibrated hot

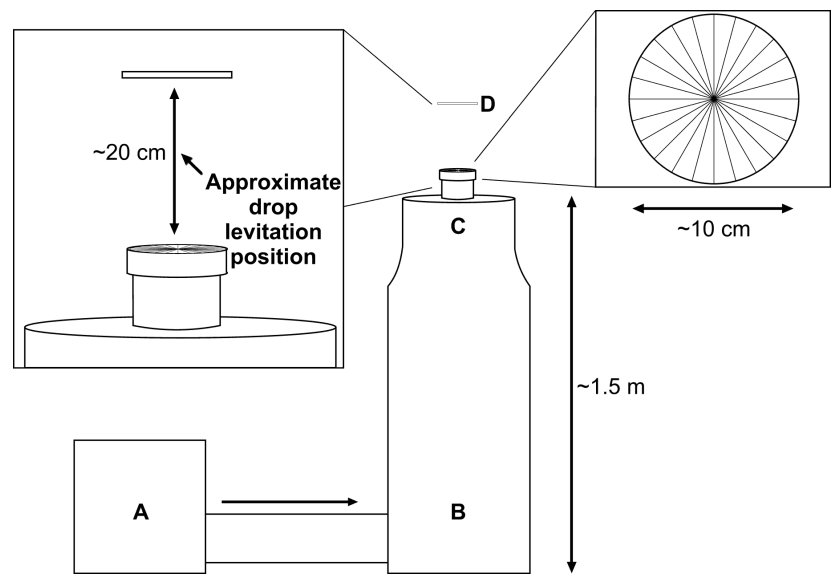

Fig. 1. Experimental setup; vertical wind tunnel: fan (A) blows air through into settling chamber (B) to minimise turbulence before exiting through top orifice $(\mathrm{C})$. Orifice is covered with a wire grate to produce a radial pressure profile which in conjunction with top plate (D) provides a back-pressure and creates a stagnation well in which drops can levitate.

wire probe to be $10.4 \pm 0.5 \mathrm{~m} \mathrm{~s}^{-1}$ in the centre, with an edge speed of $12.6 \pm 0.5 \mathrm{~m} \mathrm{~s}^{-1}$; the theoretical terminal velocity of drops of the size used here is $\sim 9 \mathrm{~m} \mathrm{~s}^{-1}$ (Pruppacher and Klett, 1978, Fig. 10-25, 420 pp.), indicating the air speed chosen is sufficient to allow the drop to levitate in the pressure well. The airspeed in the pressure well decreases with height and so drops oscillating within it will fluctuate in height about the position where the airspeed is equal to their terminal velocity. It is also possible to calculate the velocity shear produced: for a shear region of $10 \mathrm{~cm}$, the largest drop size used $(\sim 6 \mathrm{~mm})$, and radial air velocity difference of $2.2 \mathrm{~m} \mathrm{~s}^{-1}$, the percentage gradient in shear across the dimension of the drop is $\sim 1 \%$. Levitated drops were filmed using a high-speed camera (Photron Fastcam MC-1) at 2000 frames per second which allowed drop breakup events to be resolved confidently to approximately $1 \mathrm{~mm}$ resolution. The spatial resolution available could not be improved beyond $1 \mathrm{~mm}$ without reducing the field-of-view (required to be large enough to capture the area covered by the vertical motion of the drop), and thus an analysis of fine-scale, sub-mm drop breakup structure was not possible in this study. The higher temporal resolution available in the high speed videos allows much greater dynamical information to be extracted from the breakup process relative to past research. This may also improve the robustness of counting fragments relative to a strobe photography technique that has been used in the past. Water drops were injected from above and fell into the well. Precise control over drop-size was not possible; however, the drop impacting from above was always smaller than the levitated drop. The larger levitating drop was approximately stationary during the brief collision event and therefore at its terminal velocity. This experimental setup was dissimilar to that 
employed by Barros et al. (2008) and Low and List (1982a) where larger, faster falling drops fell from above onto smaller drops. Despite this difference, our breakup events show sufficient similarities that comparisons with other experimental observations can be made. Our lack of ability to control drop size to the same degree as other researchers prevented attempts to reproduce their fragment distributions and be able to examine whether experimental differences had a significant effect on the results. For the larger drop sizes used here, we suspect the differences in our experimental setup may not have a significant effect given the breakup type similarities observed (particularly for filament and sheet breakup). We encourage the reader to view the associated video data available online at http://youtu.be/3lxOFufnQZg.

In this short investigation, a total of only 25 collisional drop breakup events were examined. Failed breakup events were not counted given the different physical system. Resulting breakup fragments were counted by eye from the video data, and binned to $1 \mathrm{~mm}$ size; resolution limitations prevented higher accuracy. Note thus, that all particles $1 \mathrm{~mm}$ or smaller were binned as $1 \mathrm{~mm}$.

\section{Results and discussion}

\subsection{Experimental observations and simulated breakup distribution comparisons}

While other researchers have observed 4 distinct types of breakup - filament (neck), sheet, disc and bag (see Sect. 1 for definitions) - we only observed three, namely filament, sheet and bag, and this is likely a consequence of the use of larger drops. Based on our observations (http://youtu.be/ 31xOFufnQZg), we define the breakup types observed below.

\section{Filament breakup}

After collision, the drops briefly coalesce and then separate. The original drop sizes are often approximately restored and an interconnecting bridge between them forms before disintegrating into a spectrum of smaller drops (Fig. 2i). The disintegration of the filament bridge tends to depend on the thickness variations along its length; the thicker parts typically form larger drops as the filament destabilises and pinches during drop formation. Other researchers have observed that filament breakup is associated with glancing collisions. However, we observed that it could sometimes occur with any nature of drop collision; after collision, the larger drops used here can become sufficiently unstable from the impact that either immediately upon collision or at a variable time $(<1 \mathrm{~s})$ afterward, the drop's configuration becomes conducive to the filament type breakup.

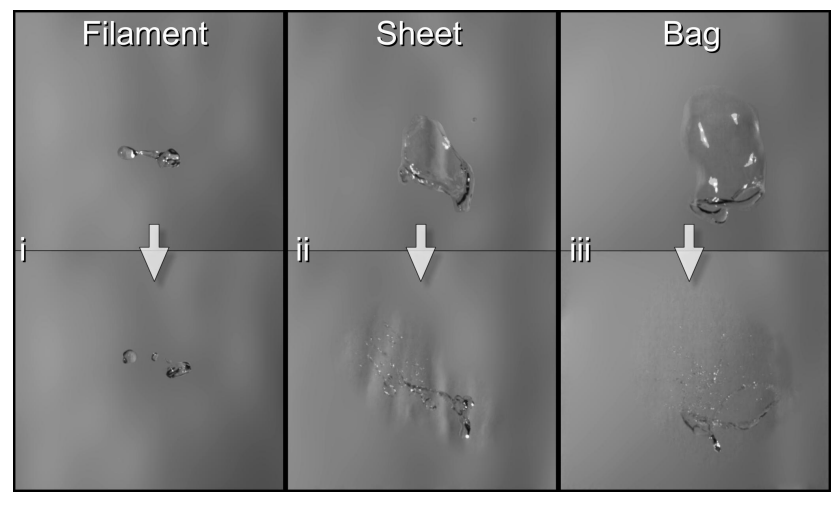

Fig. 2. Images of observed drop breakup types.

\section{Sheet breakup}

After collision, the resulting coalesced drop tends to flatten out roughly horizontally during oscillations, before lifting at one edge and disintegrating into many smaller drops (Fig. 2ii). Other researchers found that sheet breakup was associated with a more direct collision nearer the edge than the centre, to cause one side to flatten out before disintegrating. The sheet breakups we observed tended to be more associated with the larger resulting coalesced drop entering a flattened-out configuration some variable time length $(<1 \mathrm{~s})$ after collision-coalescence due to the oscillations setup by the impact.

\section{Bag breakup}

Bag breakups are similar to sheets initially; after coalescence, the resulting oscillating drop can flatten out some time $(<1 \mathrm{~s})$ after impact. However, the drop's centre, rather than edge, can lift and arch in the air stream to form a thinning bag, edged by the drop rim containing the bulk of the water (Fig. 2iii). This bag rapidly expands upwards before bursting explosively to produce numerous smaller drops. The size of the bag and its inflation depth varied widely and were likely related to the thickness of the initially arching drop centre. This mode of breakup has rarely been observed by other researchers (Low and List only saw 3 such events in 761 breakups) and the reason we saw so many is likely due to the larger drop sizes used here. It is worth noting that we did not see any disc breakups - probably because bag breakups are more preferential at larger parent drop sizes, and both the original drops were large in size.

To try and quantify how the drop interactions in our experiments may differ energetically from that of other researchers, we calculated the collisional kinetic energy that the drop-pair interaction would have had under natural free-fall conditions (using Eq. (3.1) in Low and List, 1982a). These values were within an order of magnitude of the collisional kinetic energy estimated in our observations, to the accuracy of our 
Table 1. Drop breakup data, including initial and coalesced drop sizes. The large drop was approximately stationary during collision event; smaller drop velocity was determined from video footage. Theoretical collisional kinetic energy calculated for equivalent natural drop-pair collision using Low and List (1982b).

\begin{tabular}{|c|c|c|c|c|c|c|c|c|c|c|c|c|c|c|}
\hline & \multirow[t]{2}{*}{$\begin{array}{l}\text { Breakup } \\
\text { mode }\end{array}$} & \multirow[t]{2}{*}{$\begin{array}{r}\text { small drop } \\
\text { velocity } \\
\left(\mathrm{m} \mathrm{s}^{-1}\right)\end{array}$} & \multirow[t]{2}{*}{$\begin{array}{r}\text { small } \\
\text { drop-size } \\
(\mathrm{mm})\end{array}$} & \multirow[t]{2}{*}{$\begin{array}{r}\text { large } \\
\text { drop-size } \\
(\mathrm{mm})\end{array}$} & \multirow[t]{2}{*}{$\begin{array}{l}\text { Size } \\
\text { ratio }\end{array}$} & \multirow[t]{2}{*}{$\begin{array}{r}\text { Coalesced } \\
\text { drop-size } \\
(\mathrm{mm})\end{array}$} & \multirow[t]{2}{*}{$\begin{array}{r}\text { Collisional } \\
\text { kinetic energy } \\
(\mathrm{CKE})(\mu \mathrm{J})\end{array}$} & \multirow[t]{2}{*}{$\begin{array}{r}\text { Theoretical } \\
\text { CKE } \\
(\mu \mathrm{J})\end{array}$} & \multicolumn{6}{|c|}{ Bin size $(\mathrm{mm}) \&$ count } \\
\hline & & & & & & & & & 1 & 2 & 3 & 4 & 5 & 6 \\
\hline 1 & Bag & 0.50 & 3 & 5 & 1.7 & 5 & 1.8 & 6.5 & 44 & 6 & 2 & 1 & 0 & 0 \\
\hline 2 & & 0.67 & 4 & 5 & 1.3 & 6 & 7.4 & 0.9 & 18 & 6 & 3 & 1 & 0 & 0 \\
\hline 3 & & 0.67 & 3 & 5 & 1.7 & 6 & 3.1 & 6.5 & 63 & 10 & 4 & 1 & 0 & 0 \\
\hline 4 & & 0.33 & 3 & 6 & 2.0 & 6 & 0.8 & 7.6 & 73 & 5 & 4 & 1 & 0 & 0 \\
\hline 5 & & 0.67 & 4 & 4 & 1.0 & 5 & 7.4 & 0.0 & 20 & 1 & 3 & 1 & 0 & 0 \\
\hline 6 & & 0.67 & 4 & 6 & 1.5 & 7 & 7.4 & 1.3 & 53 & 4 & 3 & 1 & 1 & 0 \\
\hline 7 & & 0.67 & 4 & 6 & 1.5 & 6 & 7.4 & 1.3 & 48 & 10 & 2 & 2 & 0 & 0 \\
\hline 8 & & 0.50 & 4 & 6 & 1.5 & 7 & 4.2 & 1.3 & 60 & 16 & 3 & 0 & 2 & 0 \\
\hline 9 & & 0.50 & 4 & 6 & 1.5 & 7 & 4.2 & 1.3 & 11 & 2 & 2 & 4 & 0 & 0 \\
\hline 10 & & 0.67 & 4 & 5 & 1.3 & 6 & 7.4 & 0.9 & 35 & 11 & 3 & 1 & 0 & 0 \\
\hline 11 & & 0.67 & 4 & 7 & 1.8 & 7 & 7.4 & 1.4 & 38 & 15 & 4 & 2 & 1 & 0 \\
\hline 12 & & 0.25 & 4 & 6 & 1.5 & 7 & 1.0 & 1.3 & 27 & 20 & 6 & 0 & 1 & 0 \\
\hline 13 & & 0.67 & 4 & 5 & 1.3 & 6 & 7.4 & 0.9 & 52 & 3 & 6 & 1 & 0 & 0 \\
\hline 14 & Sheet & 0.40 & 4 & 6 & 1.5 & 7 & 2.7 & 1.3 & 8 & 4 & 5 & 2 & 1 & 0 \\
\hline 15 & & 0.67 & 4 & 6 & 1.5 & 7 & 7.4 & 1.3 & 2 & 4 & 2 & 1 & 0 & 1 \\
\hline 16 & & 0.50 & 4 & 5 & 1.3 & 6 & 4.2 & 0.9 & 6 & 6 & 3 & 0 & 1 & 0 \\
\hline 17 & & 0.33 & 4 & 6 & 1.5 & 6 & 1.9 & 1.3 & 34 & 9 & 5 & 1 & 0 & 0 \\
\hline 18 & Filament & 0.67 & 4 & 6 & 1.5 & 8 & 7.4 & 1.3 & 7 & 1 & 1 & 0 & 2 & 0 \\
\hline 19 & & 0.67 & 4 & 8 & 2.0 & 9 & 7.4 & 1.4 & 1 & 3 & 3 & 0 & 1 & 1 \\
\hline 20 & & 0.67 & 3 & 5 & 1.7 & 6 & 3.1 & 6.5 & 3 & 0 & 1 & 0 & 1 & 1 \\
\hline 21 & & 0.67 & 4 & 6 & 1.5 & 7 & 7.4 & 1.3 & 2 & 2 & 1 & 0 & 1 & 1 \\
\hline 22 & & 0.50 & 4 & 5 & 1.3 & 6 & 4.2 & 0.9 & 0 & 2 & 1 & 1 & 1 & 0 \\
\hline 23 & & 0.50 & 4 & 6 & 1.5 & 7 & 4.2 & 1.3 & 8 & 4 & 1 & 1 & 2 & 0 \\
\hline 24 & & 0.67 & 4 & 6 & 1.5 & 6 & 7.4 & 1.3 & 10 & 6 & 4 & 0 & 1 & 0 \\
\hline \multirow[t]{5}{*}{25} & & 0.40 & 4 & 6 & 1.5 & 7 & 2.7 & 1.3 & 2 & 3 & 0 & 1 & 0 & 1 \\
\hline & Mean & & & & & & & & & & & & & \\
\hline & Bag & 0.57 & 3.77 & 5.54 & 1.49 & 6.23 & & & 41.7 & 8.4 & 3.5 & 1.2 & 0.4 & 0.0 \\
\hline & Sheet & 0.48 & 4.00 & 5.75 & 1.44 & 6.50 & & & 12.5 & 5.8 & 3.8 & 1.0 & 0.5 & 0.3 \\
\hline & Filament & 0.59 & 3.88 & 6.00 & 1.55 & 7.00 & & & 4.1 & 2.6 & 1.5 & 0.4 & 1.1 & 0.5 \\
\hline
\end{tabular}

measurements (Table 1). Thus, our physical situation is energetically similar to that in nature despite the apparent dynamical differences. The observations we made question the direct importance of collisional kinetic energy to the resulting breakup drop-size distribution in our experiments. Low and List (1982a) suggested collisional kinetic energy was important in determining the likelihood of breakup. We observed a variation in oscillation behaviour and lengths of time before breakup after a collision-coalescence event despite some similar pre-collision conditions. In addition to the impact, interaction with the air is likely to have contributed to these drop oscillations for the larger drops used. Yet after coalescence, once the drop configured itself where it became apparent thata given breakup mode will occur in the video data, the resulting fragment drops for that breakup type followed a similar distribution on average. Our observations suggest that either collisional kinetic energy or earlier drop interaction history may not necessarily be directly important to the resulting post-breakup fragment distributions in our experimental setup, and that they may only be indirectly important through possibly influencing the eventual breakup type - particularly for smaller drop sizes. Only the breakup type itself seems to directly determine the average resulting drop-size distribution.

Of the 25 drop-breakup events observed here, 8 were filaments, 4 were sheets, and 13 were bags. The experimental data is summarised in Table 1 , and the resulting drop size distributions are shown in Fig. 3. In addition, fragment size distributions were also computed using the parameterisations given by Low and List (1982b), with the corrections reported in List et al. (1987), and using parameterisations by McFarquhar (2004a). The Low and List (1982b) and McFarquhar (2004a) parameterisations can be used to calculate the fragment distribution due to breakup in 3 different breakup types: disk, sheet, and filament. For filament breakup it is assumed that two primary modes result; one centred on the 


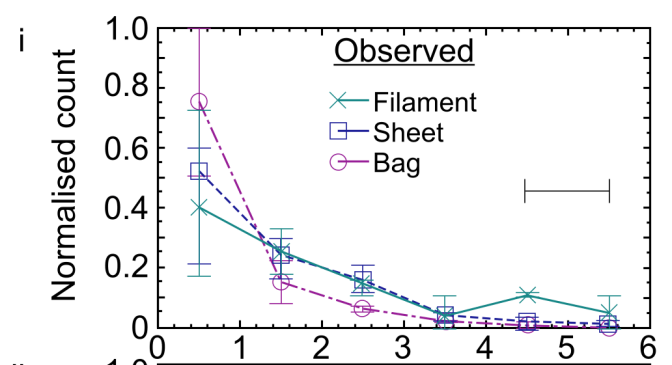

ii
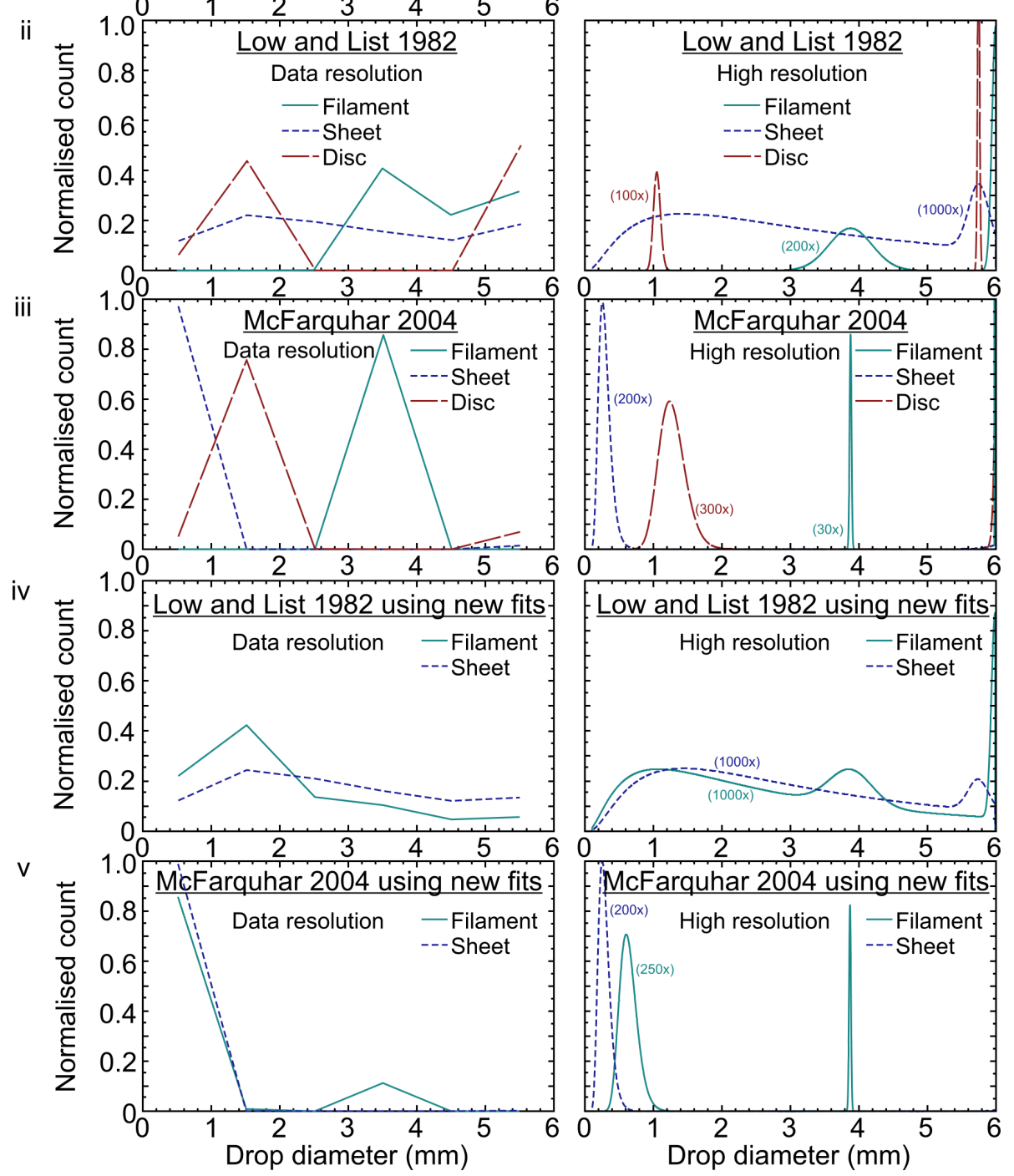

Fig. 3. Normalised histogram comparisons between observed drop breakup distribution and those predicted by the Low and List (1982a, b) and McFarquhar (2004a) parameterisations for each breakup type. Bin values represent lower limit sizes; a drop diameter of " 0 " represents all particles between 0 and $1 \mathrm{~mm}$, and so forth. Interquartile range is shown in observed results; horizontal bar in plot (i) represents what is deemed to be the maximum error in the drop-size. Both data resolution $(1 \mathrm{~mm})$ and high resolution $(1 \mu \mathrm{m})$ are shown.

small drop diameter and the other on the coalesced drop diameter; in the case of Low and List these are normally distributed whereas in the case of McFarquhar the small mode is normally distributed, but the large mode is a delta function of size equal to the coalesced drop size. In addition it is assumed that a third mode results through disintegration of the adjoining bridge between the two primary drops. This mode is assumed to be log-normally distributed in both the Low and List, and McFarquhar methods. For sheet breakup, two modes are assumed to result, a normally distributed mode centred on the large drop and a log-normally distributed, this is the same for disk break-up. Common to both the Low and 
List and McFarquhar method is the way in which the number of resulting drops from the each of the break-up types are determined, which is defined in by Low and List (1982b). The mode diameters and relative heights of all of the modes comprising the break-up functions for filament, sheet and disk are determined in different ways for the two parameterisations (see original papers for details). Once the parameters of each distribution are known they are integrated over the limits of the size bins used in this observed data (1 mm width) to enable fair comparison. We were not required to calculate the total breakup function (as in Low and List, 1982b) since we were able to classify each observed breakup event into the one of the three types.

Observed filament and sheet breakups are compared with those derived from the parameterisations. We did not observe disc breakups to enable comparison, and there is no data available to us that we are aware of in the literature to compare our bag breakups with.

The observed breakup distributions (Fig 3i) reveal a consistent pattern of increasing drop concentration with decreasing drop-size for a given breakup type. The concentration of smaller drops (1 $\mathrm{mm}$ or less in diameter) increases as breakup type transitions from filament ( $\sim 40 \%$ of total), through sheet ( $\sim 50 \%)$, to bag breakup ( $\sim 75 \%)$, where explosive breakup was more likely to be observed. This trend is qualitatively consistent with the experimental observations of other researchers (e.g. Barros et al., 2008) despite their alternative experimental setup (see Sect. 2). Sheet and bag breakup produced relatively fewer larger drops (1 $\mathrm{mm}$ or larger) than filament breakup, as would be expected from a greater portion of the coalesced drop mass transferring to predominantly larger concentrations of smaller drops on breakup for those two breakup types. Sheet breakup had slightly higher concentrations of drops $2-3 \mathrm{~mm}$ in diameter than bag breakup, consistent with the reduced breakup explosiveness. The interquartile range in the observed distributions was greatest for smaller drop sizes for all breakup types; the number of small drops produced was highly variable for a particular breakup event. Larger drops were more consistent in concentration with the exception of filament breakup, whose propensity to produce larger drops more frequently led to greater variability between breakup events.

In the computed parameterised distributions (Fig. 3ii and iii), two resolutions of data are presented. The first is data resolution corresponding to a bin size of $1 \mathrm{~mm}$ - the minimum size used in the observations - to help with direct comparisons. The second, higher resolution of $1 \mu \mathrm{m}$ is used to show the finer details of the parameterisations. The higher resolution data had to be normalised; the scaling factors for filament, sheet and disc breakup lines were 200, 1000 and 100 respectively for the Low and List parameterisations, and 30, 200 and 300 respectively for the McFarquhar parameterisations.
For the drop sizes we interacted experimentally, the computed distributions using the Low and List parameterisations (Fig. 3ii) showed key differences to our observed data. Comparing filament breakup, the parameterisations over-predict the number of resulting drops that are $3 \mathrm{~mm}$ or larger, but under-predict the number of drops $2 \mathrm{~mm}$ or smaller, particularly for the smallest sizes. The parameterisations predict no drops of these small sizes despite the fact that we observed them to be most numerous despite our relative insensitivity to these smaller sizes due to resolution restrictions. The predicted distribution also reveals a mode at the largest drop-size associated with the larger parent drops which is not always present in the observations. Predicted sheet fragment distributions show broad agreement in concentration for fragment sizes between $2-5 \mathrm{~mm}$, with decreasing concentration for larger fragments. The parameterisations over-predict the number of resulting drops, however, as fragment size increases above $5 \mathrm{~mm}$. At the smallest fragment drop-size, the parameterisations suggest decreasing concentration, and at the largest sizes, predict increasing concentration which is the opposite of the observations. Comparisons of the observations to the predicted fragments indicate that there is enough similarity to be able to cautiously compare the sheet breakups we observed with those of other researchers who used a different experimental approach.

It is possible that these discrepancies result from the Low and List parameterisations being less applicable to the relatively larger drop-pair sizes interacted in these experiments, ranging in size from 3-6 mm with size ratios of 1.0-2.0 (Table 1); those used by Low and List (1982a) ranged in size from $0.395-4.600 \mathrm{~mm}$ and had size ratios between 1.8-11.4. In general, the parameterisations of Low and List may only have application to the drop-pair sizes used in the experiments from which they were constructed (larger size ratios are, however, likely to be more representative of breakup events inside natural clouds, McFarquhar and List, 1991b). This is further supported by two further notable differences in the filament breakup distributions in Fig. 3i: (a) the presence of a saddle between the two observed distribution modes; (b) the observed shifting to a smaller size of the mode corresponding to the larger of the original drops. The saddle, (a), is possibly a consequence of how the filament bridge forms between drop-pairs of such large size; in the case observed here with two larger drops 4-6 $\mathrm{mm}$ in diameter, the bridge that formed was often substantial, containing a greater water mass. This often allowed greater filament length before breaking into smaller drops, and thus led to greater numbers of smaller drops, to form a more substantial mode at smaller sizes. A shorter bridge would more likely be formed by smaller parent drops, and result in less beading and reduced smaller drop concentration, and distributions more comparable to those described by the Low and List parameterisations. Furthermore, because the drops used here where larger than have been used previously, it is more likely that they will be separated in size in the resulting distribution from the smaller 
fragments of the bridge. The modal size shift, (b), is likely a consequence of mass conservation; the second mode at larger size corresponds to a size slightly smaller than the parent drop sizes. In filament breakup, the parent drops are typically restored, with mass lost to form the filament bridge; the corresponding reduction in drop-size is represented by this second mode. No such correspondence between secondary mode and parent drop-size is observed by the Low and List parameterisations; the second mode remains centred on the larger drop-size after breakup. This may suggest that conservation of mass requires a more complete treatment in breakup parameterisations. McFarquhar (2004a) constructed new parameterisations which treated this explicitly. The parameterisations, however, assumed that the mass in the filament was not contributed by the larger of the two parent drops (modelled as a delta function) - based on analysis of the Low and List parameterisations by Brown (1997) - and so the modal shift observed in experimental data is not represented, as seen in Fig. 3iii. However, in the experimental observations of other researchers, filament breakup has been associated with mostly glancing impact between the two parent drops which may result in little modal shift. In our experiments, most of our drops coalesced before breaking up which is more likely to result in greater mass being contributed to the filament than with a glancing edge impact, exaggerating any modal shift. Furthermore, the McFarquhar parameterisations reveal that neither filament nor sheet breakup distributions compares as well to our observed data relative to the Low and List parameterisations when extrapolated to large drop sizes. Larger numbers of smaller drops are predicted for sheet breakup; however, this seems to be a significant over-estimate.

In examining how well mathematical fits to the original observed fragment data collected by Low and List (1982a) scaled to drop sizes outside the parameter space to which they were originally compared, we noted areas where the fits broke down. Equation (3.3) given in Low and List (1982b) describes the number of fragments for filament breakup; however this approximation to the observed data produces negative values for the larger drop sizes interacted in our experiments, in which we saw many smaller drops (note that Low and List do account for this by ensuring that the minimum value this equation can give is 2 to remain physically sensible). By summing the average numbers of fragment drops in each distribution bin for filament breakup, we were able to acquire an equivalent fragment number value (equal to 10.2) that is obtained using Eq. (3.3) in the Low and List approximation. Plotting this value amongst the others provided by Low and List against the difference in drop sizes revealed families of straight lines through the origin, with a gradient depending on the ratio of the small to large drop-size. Using this empirical observation, we formulated Eq. (1) (note the final term is to ensure that in the case where $D_{\mathrm{S}}=D_{\mathrm{L}}$, there are at least the original 2 parent drops resulting). Using non-linear regression using least squares min- imisation, $\alpha$ was calculated to be 55.8646. Repeating this process for sheet breakup, we formulated Eq. (2), where $\gamma=118.0340$ and $\beta=3.1243$. These approximations also fit the fragment distributions observed by Low and List reasonably well as shown in Fig. 4.

$$
\begin{aligned}
& F_{\mathrm{f}}=\alpha\left(\frac{D_{\mathrm{S}}}{D_{\mathrm{L}}}\right)\left(D_{\mathrm{L}}-D_{\mathrm{S}}\right)+2 \\
& F_{\mathrm{S}}=\gamma D_{\mathrm{L}}^{\beta}+2
\end{aligned}
$$

Using these equations in lieu of those derived by Low and List in their parameterisations and those of McFarquhar (2004a), we recalculated the predicted fragment distributions for the larger drop sizes used in our experiments; these are plotted in Fig. 3iv and $\mathrm{v}$ respectively (note for high resolution plots, normalisation scaling factors of 1000 for both sheets and filaments were applied to the Low and List parameterisations, and 250 and 200 for filament and sheet respectively for the McFarquhar parameterisations so that features can be identified on the same axis scale). The new fragment fit increases the concentration of drops from 1-3 mm and decreases the concentration of drops $3-5 \mathrm{~mm}$, relative to the original Low and List filament breakup parameterisations, to be considerably closer to those observed (Fig. 3iv). Sheet breakup fragments are not significantly altered. The new approximation still does not capture the larger number of smaller drops observed, and for filament breakup, this could be related to the way the standard deviation of the lognormal part of the distribution is calculated - it may be overestimated by Low and List and underestimated by McFarquhar. Fragment concentration for filament breakup using the alternate fits in the McFarquhar parameterisations, increases for sub$\mathrm{mm}$ drops to larger values than is observed and decreases for sizes 3-4 mm to values comparable to those observed. Sheet breakup fragments are not significantly altered. In general, the original Low and List parameterisations with adjusted fragment concentration approximations compare better with the observed concentrations for all but the smallest fragment sizes, but still do not reproduce the saddle and mode shifts observed for filament breakup. Further work is suggested to improve parameterisations to fit breakup distributions of all observed drop sizes.

In addition to 25 coalescence and breakup events listed in Table 1, we observed separately an instance where a near-filament breakup event failed and the drop did not breakup, but re-formed into a flattened drop before entering bag breakup within a few hundred milliseconds. We also observed two instances of breakup occurring twice in succession from the same parent drop $(<1 \mathrm{~s})$ : the first involved a filament breakup followed by a bag breakup of the larger remaining drop; the second involved two bag breakups where after the first breakup, a large enough drop remained to break up again as a secondary bag breakup event. This type of double breakup is not currently modelled when applying 


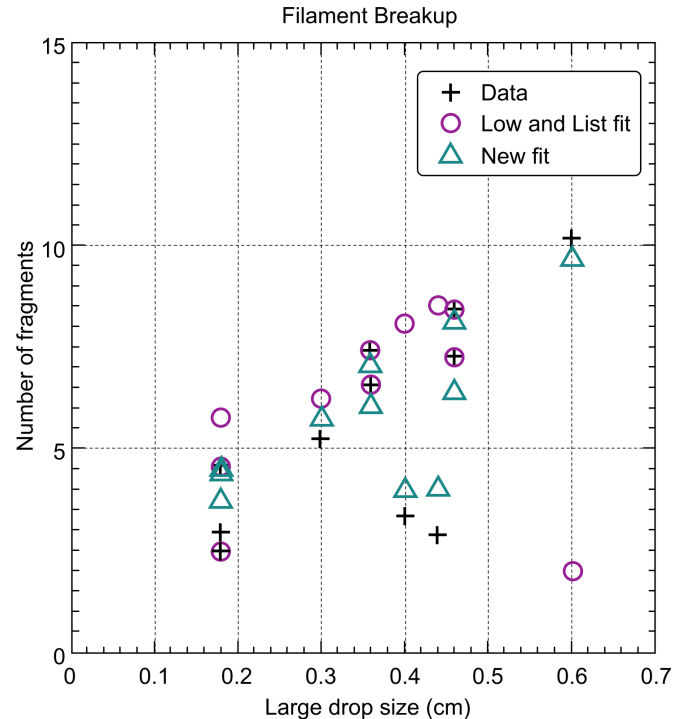

ii

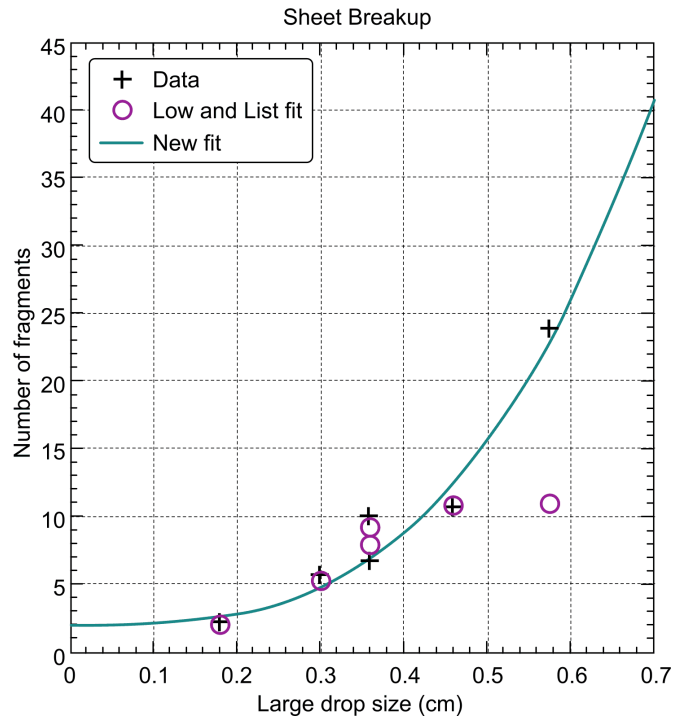

Fig. 4. Comparison between the original Low and List (1982a) data combined with our data, and both the original Low and List fits and our modified fits.

the Low and List breakup parameterisation to the stochastic coalescence and breakup equation; however, it is difficult to judge the statistical relevance of this type of event in nature. Given that for our experimental setup we were able to observe that a variable length of time can pass between collision-coalescence and eventual breakup $(<1 \mathrm{~s})$, it is worth highlighting the question of how long is required before breakup for the event to be classified as collisionallyinduced or spontaneous. In very few of the preliminary experiments separate to the data presented here, true spontaneous breakup was observed where a large drop $(>6 \mathrm{~mm})$ in the pressure-well of the wind tunnel that had not been subjected to direct collisions, eventually broke up after tens of seconds. The variable length of time between collision- coalescence and breakup is considerably shorter $(<1 \mathrm{~s})$ than the timescales associated with spontaneous breakup $(\gg 1 \mathrm{~s})$.

\subsection{Simulation of stochastic coalescence and breakup}

The evolution through coalescence and breakup of an initial drop spectrum was simulated through the solution of the stochastic coalescence and breakup equation. Our method to solve the stochastic coalescence/breakup equation follows Bott (1998). Bott has demonstrated that this method gives excellent results when compared to the analytical solution to Golovin's kernel and when compared to the more computationally expensive Berry and Reinhart method. Also, it has been shown that a 1-moment approach is adequate for resolving stochastic breakup (Feingold et al., 1988).

A mass grid is used, of which each adjacent bin is $2^{1 / 2}$ times the previous bin. The number-size distribution is transformed into a mass distribution following Berry and Reinhart and the equation is integrated using a simple time-stepping scheme. Firstly the loss of mass from two bins containing colliding drops is calculated using the collision kernel of Long (1974). The mass from these interacting bins is added together and the fraction that coalesces is added to the bin that is nearest to the sum of the two interacting bins - of which a fraction is then transported to the adjacent mass bin using the exponential flux method. The mass that does not coalesce is redistributed on the mass grid using the fragment distributions calculated from Low and List (1982b). Also implemented are spontaneous breakup schemes where drops of a certain size have a finite probability of breaking up due to hydrodynamic instability, yielding a fragment distribution that is exponentially distributed (see Kamra et al., 1991; Komabayasi et al., 1964; Villermaux and Bossa, 2009).

Cloud drop-size distribution evolution was simulated for $1500 \mathrm{~s}$ with the spontaneous breakup scheme active in all cases to examine how drop mass-weighted mean diameter (Fig. 5a) and concentration (Fig. 5b) altered. Initial cloud drop concentration and cloud liquid water content were varied for cases when collisional breakup was active and inactive to test sensitivity. The resulting drop size distributions are shown in Fig. 6.

Figure 5 reveals that the time to reach a steady-state in mean drop-size and concentration is heavily influenced by the cloud liquid water content, with greater values allowing steady-state to be reached earlier; a value of $3 \times 10^{-3} \mathrm{~kg} \mathrm{~kg}^{-1}$ allows steady-state to be reached by $\sim 500 \mathrm{~s}$, and $1 \times 10^{-3} \mathrm{~kg} \mathrm{~kg}^{-1}$ by $\sim 1500 \mathrm{~s}$. Collisional breakup reduces the resulting steady-state mean drop diameter from $\sim 2.5 \mathrm{~mm}$ to $\sim 0.5 \mathrm{~mm}$ ( $\sim$ factor of 5), which is not substantially changed by initial cloud liquid water content. Similarly, steady-state drop concentration was increased by collisional breakup from $\sim 1.5 \times 10^{3} \mathrm{~m}^{-3}$ to $\sim 7 \times 10^{4} \mathrm{~m}^{-3}$ ( $\sim$ factor of 50$)$. The resulting drop size distributions (Fig. 6) did not show a three-peaked distribution as observed in modelling studies by List and McFarquhar (1990) and 

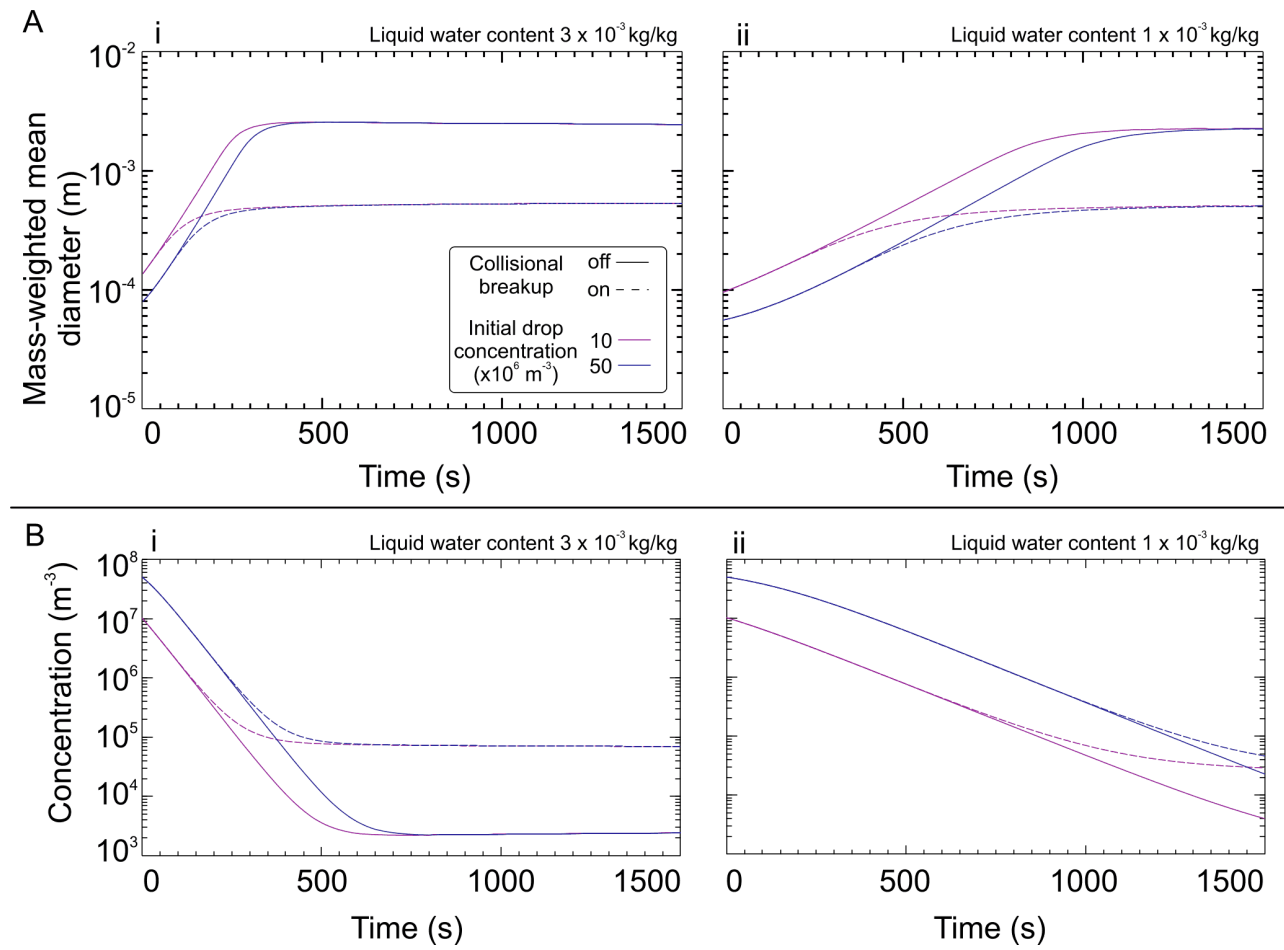

Fig. 5. Simulations of mass-weighted mean diameter and concentration changes during cloud evolution for $1500 \mathrm{~s}$. Initial drop concentration and cloud liquid water content were varied for cases when collisional breakup was either active or inactive. Spontaneous breakup was active in all runs.

McFarquhar and List (1991a, b) in which a requirement for such distributions appeared to be the presence of overlapping and interacting falling rain pulses. The initial drop concentration had less effect on the time taken to reach steady-state; a factor of 5 increase in initial drop concentration led to a delay of $\sim 100$ s to reach steady-state. It also had no effect on the resulting mean size or resulting drop concentration; however, increasing initial drop concentrations resulting in more rapid mean size and concentration changes as steadystate is approached - this follows from the reduced initial concentration that increasing initial drop number causes for a fixed initial cloud liquid water content. This rate of change is affected to a considerably greater extent from alterations in initial cloud liquid water content however.

The resulting drop size distributions (Fig. 6) show no presence of a three-peaked distribution. Increasing initial cloud liquid water content increases the maximum drop-size by $0.5 \mathrm{~mm}$ to $\sim 3 \mathrm{~mm}$ when collisional breakup is active. In the absence of collisional breakup, the resulting distribution is markedly broadened, particularly for greater initial cloud liquid water contents. The initial drop concentration has negligible effect on the resulting distribution regardless of whether or not collisional breakup is active. This may be relevant to considerations of how aerosols affect cloud microphysics (Lohmann and Feichter, 2005), and may also affect calculations of precipitation susceptibility (Ma et al., 2010; Stevens and Feingold, 2009). Aerosol influences on cloud drop-size distribution, cloud evolution and rain formation may be less significant relative to parameters less dependent on aerosol such as initial cloud liquid water content (for fixed drop concentration).

The distributions observed in our experiments were considered for inclusion in the computer program to solve the stochastic coalescence and breakup equation, to enable a comparison with the Low and List distributions; however, our lack of sensitivity to sizes smaller than $1 \mathrm{~mm}$ was considered a sufficient enough shortcoming to prevent this. However, it was possible to compare the estimated distribution fragment concentration from the Low and List parameterisations with those observed. From this it was possible to quantify estimated adjustments to the predicted fraction of total breakup events from the Low and List parameterisations for drop-pairs of sizes 4 and $6 \mathrm{~mm}$. These larger drop sizes are observed in nature (Hobbs and Rangno, 2004), but the Low and List parameterisations were not constructed from observations of drops of such large sizes and in fact predict very low concentrations of large $(>3 \mathrm{~mm})$ drops. Figure 7 shows both the time varying percentage fraction of total collisions, and percentage fraction of total fragments that the Low and List parameterisations predict should occur from drops of size 4 and $6 \mathrm{~mm}$ (comparable in size with the mean sizes used in the experiments here, Table 1) for two high values of cloud liquid water content of 3 and $5 \mathrm{~kg} \mathrm{~kg}^{-1}$. It is assumed that a precipitating drop concentration of $1 \times 10^{-3} 1^{-1}$ 

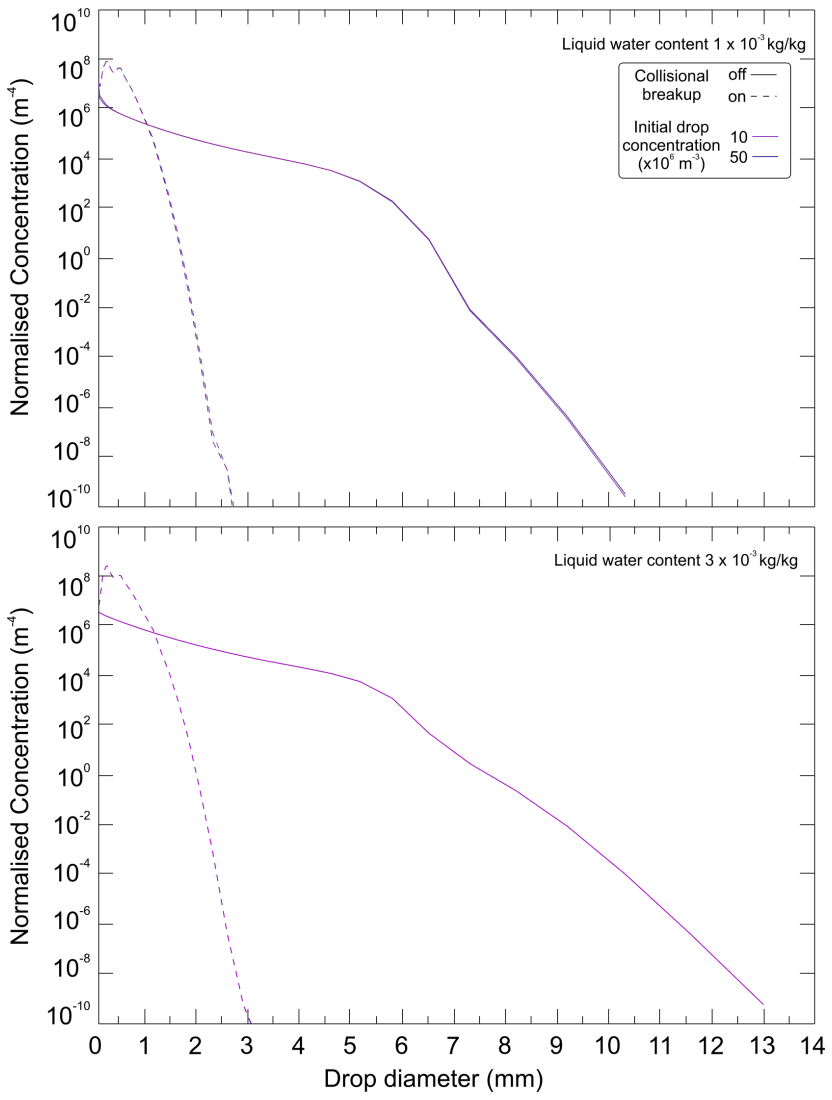

Fig. 6. Resulting simulation drop size distributions. Initial drop concentration and cloud liquid water content were varied for cases when collisional breakup was either active or inactive. Spontaneous drop breakup was active in all runs.

is present initially in the rain shaft, with an exponential dropsize distribution. Spontaneous breakup is not represented in the calculations to ensure only the collisionally-induced contributions to breakup events are represented to be consistent with the experimental observations. The Low and List parameterisations suggest that a maximum of $\sim 0.18-0.40 \%$ of total breakup events are expected to be contributed by the larger 4 and $6 \mathrm{~mm}$ drop-pair interactions $(4.0 \pm 0.5 \mathrm{~mm}$; $5.8 \pm 0.5 \mathrm{~mm})$ and $\sim 0.26-0.66 \%$ of all breakup fragments. These fractions reduce in value rapidly and are negligible by $100 \mathrm{~s}$. It is possible to compare the prediction of percentage fragment contributions with an equivalent result based on the experimental observations. By comparing the number of observed experimental breakup fragments with those estimated from the distributions resulting from the Low and List parameterisations, it is possible to find adjustment factors for each breakup type to apply to Fig. 7. These help to quantify the underestimation of fragment concentrations of the Low and List parameterisations for the drop-pair sizes used in the observations. Appendix A describes the method to establish the estimated parameterised fragment concentrations used to determine these factors, and these are listed in Table 2. Rela-

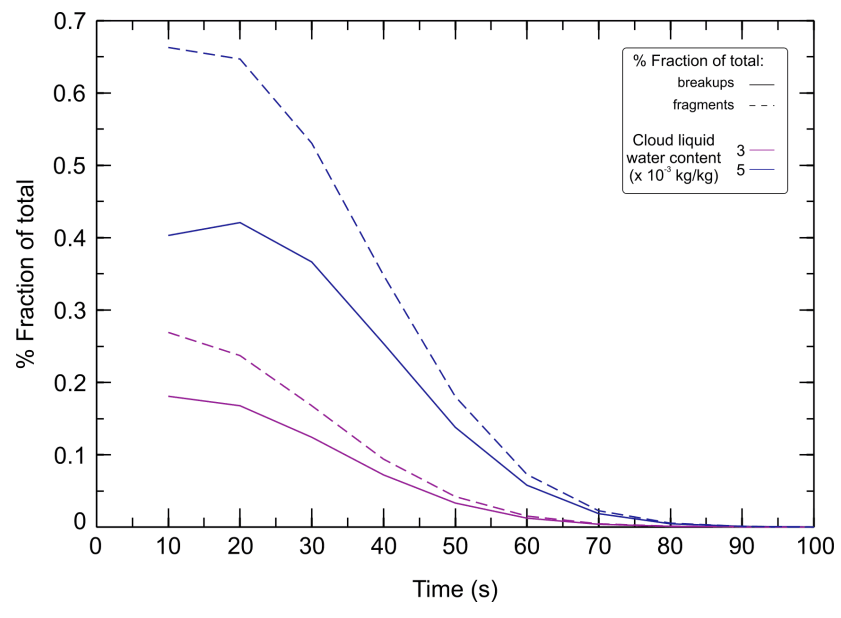

Fig. 7. The percentage fraction of total breakup events (solid lines) and percentage fraction of total resulting fragments (dashed lines) produced by a 4 and $6 \mathrm{~mm}$ drop-pair relative to all drop interactions. Cloud liquid water content was varied between large values of $3 \mathrm{~kg} \mathrm{~kg}^{-1}$ (pink) and $5 \mathrm{~kg} \mathrm{~kg}^{-1}$ (blue).

tive to the values observed experimentally, there is a factor of $\sim 4.2$ fewer total fragments predicted for filament and sheet breakup, and a factor of $\sim 6$ fewer for disc breakup. Note that disc and sheet predictions are both compared to our observed sheet breakup data; it should be noted that our sheet data does not match well with the predicted disc data and so values here for disc breakup should be treated cautiously. After adjustment of the maximum percentage contributing fractions in Fig. 7 , as many as $\sim 1-3 \%$ of total fragment drops could actually be contributed by the larger drop-pair interactions for filament and sheet breakup.

\section{Summary}

The breakup of large colliding water drops has been examined using a vertical wind tunnel and high speed video. The objectives of this study were to examine the breakup fragment distributions of larger drops than have been used in previous experimental studies. The conditions which lead to breakup of a given type were not examined. Additionally, the breakup distributions were compared to predicted distributions from computed extrapolations of the parameterisations of Low and List (1982b) and McFarquhar (2004a) to examine whether they are valid for drop sizes larger than those originally used in their construction. Furthermore, simulations of cloud drop-size distribution evolution and contributions to this from larger drop-pair sizes are performed to examine the significance of these interactions.

Of the 25 drop-breakup events observed here, 8 were filaments, 4 were sheets, and 13 were bags (Table 1). The concentration of smaller fragment drops ( $1 \mathrm{~mm}$ or less in diameter) in our observations increases as breakup type transitions 
Table 2. Factors to adjust the predicted percentage fraction of total resulting breakup fragments contributed by 4 and $6 \mathrm{~mm}$ drop-pair interactions based on the Low and List parameterisations. The adjusted range corresponds to initial cloud liquid water contents of 3 and $5 \mathrm{~kg} \mathrm{~kg}^{-1}$. (See Appendix A for procedure to calculate $k$ value and total estimated fragments.) Note that disc and sheet predictions are both compared to our observed sheet breakup data (see discussion section).

\begin{tabular}{llcccc}
\hline $\begin{array}{l}\text { Breakup } \\
\text { type }\end{array}$ & $k$ value & $\begin{array}{c}\text { Total } \\
\text { estimated } \\
\text { fragments }\end{array}$ & $\begin{array}{c}\text { Observed } \\
\text { average total } \\
\text { fragments }\end{array}$ & $\begin{array}{c}\text { Relative } \\
\text { fractional } \\
\text { difference }\end{array}$ & $\begin{array}{c}\text { Adjusted maximum \% } \\
\text { fraction contribution } \\
\text { of fragments }\end{array}$ \\
\hline Filament & $1.64 \times 10^{-4}$ & 2.46 & 10.25 & 4.17 & $1.1-2.8$ \\
Sheet & $5.64 \times 10^{-5}$ & 5.66 & 23.75 & 4.20 & $1.1-2.8$ \\
Disc & $2.58 \times 10^{-4}$ & 3.87 & 23.75 & 6.14 & $1.7-4.1$ \\
\hline
\end{tabular}

from filament $(\sim 40 \%$ of total), through sheet $(\sim 50 \%)$, to bag breakup ( $75 \%)$ (Fig. 3). Fragment distributions derived from the Low and List parameterisations tended to over-predict the number of large drops, but under-predict the number of drops $2 \mathrm{~mm}$ or smaller, particularly for the smallest sizes (Fig. 3ii). The McFarquhar parameterisations do not compare well with the observed fragment distributions when extrapolated to the larger drop sizes used here (Fig. 3iii). Using new approximations for resulting fragment concentrations (Eqs. 1 and 2) based on empirical observations from the combination of Low and List's experimental drop breakup data with ours, new fragment distributions were calculated for the parameterisations of both Low and List, and McFarquhar, which satisfied both datasets reasonably well (Fig. 3iv and v; further details in Sect. 3.1, paragraph 11). However, the new approximation still did not capture the larger number of smaller drops observed. Further work is suggested to improve parameterisations to fit breakup distributions of all observed drop sizes.

The evolution of a simulated initial drop distribution was computed through the solution of the stochastic coalescence and breakup equation (Sect. 3.2). The computations revealed that the time for mean drop-size and drop concentration steady-state to be reached was independent of whether collisional breakup was active, was weakly influenced by initial drop number, but was heavily dependent on initial cloud liquid water content (Figs. 5, 6 and Sect. 3.2). The resulting drop spectrum (Fig. 6) was again influenced heavily by initial cloud liquid water content in addition to whether collisional breakup was active. The relative lack of significant influence of the initial drop concentration on the resulting steady-state mean drop diameter, drop concentration, and the resulting drop spectrum has implications when considering significance of the effect of aerosol on cloud microphysics and evolution of raindrop size distributions.

A comparison between the total number of breakup fragments from the Low and List parameterisations and those observed was made to find adjustment factors which could then be applied to the fragment contribution values given in Fig. 7. This provided an estimate for the contribution to the total fragment concentration produced by interactions between the larger 4 and $6 \mathrm{~mm}$ drops used here, based on our data. This revealed that as many as $\sim 1-3 \%$ of total fragment drops could actually be contributed by the larger drop-pair interactions for filament and sheet breakup for a precipitating drop concentration of $1 \times 10^{-3} 1^{-1}$ with an exponential drop-size distribution with cloud liquid water content ranging from $3-5 \mathrm{~kg} \mathrm{~kg}^{-1}$.

\section{Appendix A}

\section{Calculation of parameterisation adjustment factors}

In Sect. 3.2, fragment contributions from 4 and $6 \mathrm{~mm}$ drop interactions relative to all interactions are predicted based on the Low and List parameterisations and solution of the stochastic coalescence and breakup equation. By comparing the number of observed drop fragments from the estimated number suggested by the parameterisations, factors can be established for interactions between 4 and $6 \mathrm{~mm}$ drops. These can then be used to adjust the fractional contribution values suggested by the parameterisations to help better indicate their overall significance to the resulting drop size distributions, given that such large drops have been observed in natural clouds (Hobbs and Rangno, 2004).
List of terms (SI units):
$M_{\mathrm{L}} \quad$ Mass of large drop
$M_{\mathrm{S}} \quad$ Mass of small drop
$M_{\mathrm{T}} \quad$ Total mass
$M_{i} \quad$ Mass of distribution element
$k \quad$ Scaling factor
$\rho_{\mathrm{w}} \quad$ Density of water
$P_{i} \quad$ Drop distribution
$V_{\mathrm{L}} \quad$ Volume of large drop
$V_{\mathrm{S}} \quad$ Volume of small drop
$V_{i} \quad$ Volume of distribution element 
$D_{\mathrm{L}} \quad$ Diameter of large drop

$D_{\mathrm{S}} \quad$ Diameter of small drop

$D_{i} \quad$ Drop distribution fragment diameters

Considering the coalescence and breakup event, the combined masses of the two interacting drops must be equal to the mass given by the sum of the parameterised breakup distribution. This distribution is normalised and thus must have a scaling factor present to ensure it is scaled appropriately for mass, i.e. to ensure mass conservation.

$M_{\mathrm{L}}+M_{\mathrm{S}}=M_{\mathrm{T}}=k \sum_{i} M_{i} P_{i}$

Expressing mass as a product of density and volume:

$\rho_{\mathrm{w}} V_{\mathrm{L}}+\rho_{\mathrm{w}} V_{\mathrm{S}}=k \rho_{\mathrm{w}} \sum_{i} V_{i} P_{i}$

Water density terms cancel; volume can be expressed as a function of the diameter of a sphere:

$\frac{\pi}{6} D_{\mathrm{L}}^{3}+\frac{\pi}{6} D_{\mathrm{S}}^{3}=\frac{\pi}{6} k \sum_{i} D_{i}^{3} P_{i}$

Rearranging for $k$ :

$k=\frac{D_{\mathrm{L}}^{3}+D_{\mathrm{S}}^{3}}{\sum_{i} D_{i}^{3} P_{i}}$

Total particle number is thus given by:

$k \sum_{i} P_{i}$

\section{Supplementary material related to this article is available online at: http://www.atmos-chem-phys.net/11/10205/2011/ acp-11-10205-2011-supplement.zip.}

Acknowledgements. We would like to thank the EPSRC instrument loan pool for providing us with an opportunity to use one of their high speed cameras for this research. We would also like to thank Tom Choularton for guidance and comment during the project. Part of this work was supported by the Natural Environment Research Council [grant number NE/G000875/1].

Edited by: M. Petters

\section{References}

Barros, A. P., Prat, O. P., Shrestha, P., Testik, F. Y., and Bliven, L. F.: Revisiting Low and List (1982): Evaluation of Raindrop Collision Parameterizations Using Laboratory Observations and Modeling, J. Atmos. Sci., 65, 2983-2993, doi:10.1175/2008JAS2630.1, 2008.

Beard, K. V., Johnson, D. B., and Baumgardner, D.: Aircraft Observations of Large Raindrops in Warm, Shallow, Convective Clouds, Geophys. Res. Lett., 13, 991-994, 1986.
Bott, A.: A flux method for the numerical solution of the stochastic collection equation, J. Atmos. Sci., 55, 2284-2293, 1998.

Brown, P. S.: Mass conservation considerations in analytic representation of raindrop fragment distributions, J. Atmos. Sci., 54, 1675-1687, 1997.

Debeauville, C. A., Petit, R. H., and Marion, G.: Evolution of Peaks in the Spectral Distribution of Raindrops from Warm Isolated Maritime Clouds, J. Atmos. Sci., 45, 3320-3332, 1988.

Feingold, G., Tzivion, S., and Levin, Z.: Evolution of Raindrop Spectra .1. Solution to the Stochastic Collection Breakup Equation Using the Method of Moments, J. Atmos. Sci., 45, 3387 3399, 1988.

Hobbs, P. V. and Rangno, A. L.: Super-large raindrops, Geophys. Res. Lett., 31, L13102, doi:10.1029/2004g1020167, 2004.

Joss, J. and Gori, E. G.: Shapes of Raindrop Size Distributions, J. Appl. Meteorol., 17, 1054-1061, 1978.

Kamra, A. K., Bhalwankar, R. V., and Sathe, A. B.: Spontaneous Breakup of Charged and Uncharged Water Drops Freely Suspended in a Wind-Tunnel, J. Geophys. Res.-Atmos., 96, 1715917168, 1991.

Komabayasi, M., Gonda, T., and Isono, K.: Life Time of Water Drops before Breaking and Size Distribution of Fragment Droplets, J. Meteor. Soc. Japan, 42, 330-340, 1964.

List, R. and Mcfarquhar, G. M.: The Evolution of 3-Peak Raindrop Size Distributions in One-Dimensional Shaft Models .1. SinglePulse Rain, J. Atmos. Sci., 47, 2996-3006, 1990.

List, R., Donaldson, N. R., and Stewart, R. E.: Temporal Evolution of Drop Spectra to Collisional Equilibrium in Steady and Pulsating Rain, J. Atmos. Sci., 44, 362-372, 1987.

Lohmann, U. and Feichter, J.: Global indirect aerosol effects: a review, Atmos. Chem. Phys., 5, 715-737, doi:10.5194/acp-5-7152005, 2005.

Long, A. B.: Solutions to Droplet Collection Equation for Polynomial Kernels, J. Atmos. Sci., 31, 1040-1052, 1974.

Low, T. B. and List, R.: Collision, Coalescence and Breakup of Raindrops .1. Experimentally Established Coalescence Efficiencies and Fragment Size Distributions in Breakup, J. Atmos. Sci., 39, 1591-1606, 1982a.

Low, T. B. and List, R.: Collision, Coalescence and Breakup of Raindrops .2. Parameterization of Fragment Size Distributions, J. Atmos. Sci., 39, 1607-1618, 1982 b.

Ma, X., von Salzen, K., and Cole, J.: Constraints on interactions between aerosols and clouds on a global scale from a combination of MODIS-CERES satellite data and climate simulations, Atmos. Chem. Phys., 10, 9851-9861, doi:10.5194/acp-10-98512010, 2010.

Magarvey, R. H. and Geldart, J. W.: Drop Collisions under Conditions of Free Fall, J. Atmos. Sci., 19, 107-113, 1962.

McFarquhar, G. M.: A new representation of collision-induced breakup of raindrops and its implications for the shapes of raindrop size distributions, J. Atmos. Sci., 61, 777-794, 2004a.

McFarquhar, G. M.: The effect of raindrop clustering on collisioninduced break-up of raindrops, Q. J. Roy. Meteor. Soc., 130, 2169-2190, doi:10.1256/Qj.03.98, 2004b.

McFarquhar, G. M. and List, R.: The Raindrop Mean Free-Path and Collision Rate Dependence on Rainrate for 3-Peak Equilibrium and Marshall-Palmer Distributions, J. Atmos. Sci., 48, 1999-2003, 1991a.

McFarquhar, G. M. and List, R.: The Evolution of 3-Peak Raindrop 
Size Distributions in One-Dimensional Shaft Models .2. Multiple Pulse Rain, J. Atmos. Sci., 48, 1587-1595, 1991 b.

McFarquhar, G. M. and List, R.: The Effect of Curve Fits for the Disdrometer Calibration on Raindrop Spectra, Rainfall Rate, and Radar Reflectivity, J. Appl. Meteorol., 32, 774-782, 1993.

McTaggart-Cowan, J. D. and List, R.: Collision and Breakup of Water Drops at Terminal Velocity, J. Atmos. Sci., 32, 1401-1411, 1975a.

McTaggart-Cowan, J. D. and List, R.: Acceleration System for Water Drops, J. Atmos. Sci., 32, 1395-1400, 1975 b.

Pruppacher, H. R. and Klett, J. D.: Microphysics of clouds and precipitation, D. Reidel, 1978.

Steiner, M. and Waldvogel, A.: Peaks in Raindrop Size Distributions, J. Atmos. Sci., 44, 3127-3133, 1987.
Stevens, B. and Feingold, G.: Untangling aerosol effects on clouds and precipitation in a buffered system, Nature, 461, 607-613, doi:10.1038/Nature08281, 2009.

Villermaux, E. and Bossa, B.: Single-drop fragmentation determines size distribution of raindrops, Nat. Phys., 5, 697-702, doi:10.1038/Nphys1340, 2009.

Warner, J.: Microstructure of Cumulus Cloud. I. General Features of Droplet Spectrum, J. Atmos. Sci., 26, 1049-1059, 1969.

Willis, P. T.: Functional Fits to Some Observed Drop Size Distributions and Parameterization of Rain, J. Atmos. Sci., 41, 1648$1661,1984$.

Zawadzki, I. and Antonio, M. D.: Equilibrium Raindrop Size Distributions in Tropical Rain, J. Atmos. Sci., 45, 3452-3460, 1988. 